NOVAE \& SYMBIOTIC STARS 


\title{
CLASSICAL NOVA EVOLUTION: CLUES FROM SOFT X-RAY EMISSION
}

\author{
JAMES MACDONALD \\ University of Delaware \\ Department of Physics and Astronomy, Newark, \\ DE 19716, USA
}

\begin{abstract}
Multi-wavelength observations have shown that, after optical decline, the stellar remnants of classical nova outbursts evolve at constant, near-Eddington, bolometric luminosity to high effective temperature ( $>$ $210^{5} \mathrm{~K}$ ), before turning off. Here we briefly review the observations of classical novae in this phase of evolution, and discuss what the soft X-ray observations tell us about the mass of the underlying white dwarf and the rate of mass change due to stellar winds and accretion from the stellar companion.
\end{abstract}

\section{Introduction}

A classical nova outburst occurs when a white dwarf in a close binary system accretes sufficient hydrogen-rich material from a non-degenerate companion to trigger a thermonuclear runaway (Starrfield et al. 1974). The ensuing rapid release of nuclear energy causes the photospheric luminosity to increase to greater than the Eddington limit, $L_{\text {Edd }}$, and the white dwarf envelope, of mass $M_{\text {env }}$, to expand to red giant dimensions on a dynamical time-scale, ejecting a mass, $M_{\mathrm{ej}}$, of between $10^{-6}$ and $10^{-4} \mathrm{M}_{\odot}$. Early hydrodynamic simulations found that only a fraction of the envelope is ejected in the initial explosion. The remaining envelope quickly returns to hydrostatic equilibrium and enters a steady nuclear burning phase with a constant bolometric luminosity close to $L_{\mathrm{Edd}}$.

Observationally the recognition that a constant bolometric luminosity phase is a common characteristic of classical novae followed upon a combination of ultraviolet and infrared observations of Nova FH Ser 1970 (Hyland \& Neugebauer 1970; Geisel, Kleinmann \& Low 1970; Gallagher \& Code

A. Evans and J. H. Wood (eds.), Cataclysmic Variables and Related Objects, 281-287. ( 1996 Kluwer Academic Publishers. Printed in the Netherlands. 
1974; Gallagher \& Starrfield 1976). For this moderately fast nova, the visual luminosity fell quite rapidly, with the dominance of the UV luminosity after the first week reflecting the rapidly increasing photospheric temperature. The fall in the UV after approximately $60 \ldots 80 \mathrm{~d}$ and the simultaneous emergence of the infrared is due to absorption and re-emission by dust grains in the ejecta. During the first $100 \mathrm{~d}$, the sum of UV, visual and IR luminosities were roughly constant (Gallagher \& Starrfield 1978).

Although estimates of the time to turn-off of the nuclear burning were uncertain, it was immediately clear that evolution of the stellar remnant on a purely nuclear time-scale was too slow to be consistent with the observations. Turn-off requires some mechanism of mass loss (Starrfield 1979; MacDonald, Fujimoto \& Truran 1985, hereafter MFT). In the next section, we briefly review the theory of the turn-off of classical novae. In particular, the stellar remnants are expected to evolve to high effective temperatures $\left(T_{\text {eff }}>210^{5} \mathrm{~K}\right)$ and be copious emitters of EUV/soft X-ray photons. In theory, measurement of the maximum $T_{\text {eff }}$, attained at turn-off, can be used to determine the mass of the white dwarf, and the time to reach maximum $T_{\text {eff }}$ constrains the rate of mass loss from the stellar remnant and also the rate of any accretion from the companion star. We apply these ideas to two well observed novae, GQ Mus and V1974 Cyg.

\section{The evolution to turn-off}

In discussing the evolution of the stellar remnant, it is useful to divide the nova outburst into three phases: (A) envelope expansion in response to the thermonuclear runaway, (B) common envelope evolution and (C) steady nuclear burning at constant bolometric luminosity, with possible wind mass loss or accretion from an irradiated companion.

Of crucial importance to the lifetime of phase B is the amount of envelope material remaining on the stellar remnant after phase A. Recent hydrodynamic calculations of phase A differ significantly in the fraction of envelope material that is ejected. For example, Starrfield et al. (1992), in simulations of Nova Her 1991, find $M_{\mathrm{ej}} / M_{\mathrm{env}}$ in the range $0.09 \ldots 0.67$, whereas Prialnik \& Shara (1995) find $M_{\mathrm{ej}} / M_{\mathrm{env}}$ between 0.92 and 1.00 . We suspect that this significant disagreement is due in part to Prialnik \& Shara (1995) completely suppressing convective energy transport in hydrodynamic phases of evolution and to their method of removal of mass zones that are escaping from the star (Prialnik \& Kovetz 1995). Both these effects work in the direction of increasing the amount of ejected material.

If, as the calculations of Starrfield and collaborators suggest, a significant fraction of the envelope remains after phase $A$, then the stellar remnant is larger than the orbital separation and phase B is entered. As shown by 
MacDonald (1980) and MFT, dynamical friction quickly ejects almost all of the material lying outside the orbit at about the escape speed in the vicinity of the orbit. For this phase to occur, $M_{\mathrm{A}}$, the envelope mass at the end of phase A, must satisfy (MFT)

$$
\frac{M_{\mathrm{A}}}{\mathrm{M}_{\odot}} \gtrsim 210^{-5}\left(\frac{M_{\mathrm{WD}}}{\mathrm{M}_{\odot}}\right)^{-4.5}\left(\frac{S}{10^{11} \mathrm{~cm}}\right)^{0.3}
$$

where $M_{\text {WD }}$ is the white dwarf mass and $S$ is the orbital separation. For a typical $S$ of $10^{11} \mathrm{~cm}$, this is satisfied for all the simulations of Starrfield et al. (1992). Indirect observational support for the existence of phase B in classical novae comes from comparison with very slow novae such as RR Tel and RT Ser, in which the companion is a giant star (Feast \& Glass 1974) and the orbital separation too large for a significant common envelope phase. The very slow novae have long turn-off times and, more importantly, very low expansion velocities of order $100 \mathrm{~km} \mathrm{~s}^{-1}$, whereas classical novae have expansion velocities of order the orbital velocity of the companion star or greater (MacDonald 1980). Paresce et al. (1995) find that the kinematics and morphology of the shell ejected by V1974 Cyg, determined from their HST FOC observations, are consistent with the shaping expected from a common envelope phase.

As shown by MFT, the lifetimes of phases A and B are much shorter than that of phase $\mathrm{C}$ and hence it is the lifetime of phase $\mathrm{C}$ that determines $t_{\text {rem }}$, the time for turnoff of classical nova remnant. In the absence of wind mass loss, the only process to consume envelope material is nuclear burning. On the basis of polytropic models for the envelope, MFT then estimate, for a composition representative of novae ejecta, that

$$
t_{\mathrm{rem}} \simeq 20\left(\frac{M_{\mathrm{WD}}}{\mathrm{M}_{\odot}}\right)^{-6.3} \mathrm{yr}
$$

which has a very strong dependence on white dwarf mass. However it is unrealistic to assume that the hot remnant with a luminosity close to the Eddington limit will not have a wind, whether optically thick (Bath 1978, Kato 1983, Kato \& Hachisu 1994) or line-driven (MacDonald 1984, MFT). Furthermore for systems with small orbital periods, irradiation of the companion by the hot remnant may induce accretion onto the white dwarf, prolonging phase C (Ögelman et al. 1993). If an independent estimate of $M_{\text {WD }}$ can be obtained then comparison of $t_{\text {rem }}$ with the nuclear burning time-scale in principle gives the rate of mass loss or mass gain, which can be compared to the theoretical prediction for e.g. optically thick winds. Determinations of $M_{\mathrm{WD}}$ by standard spectroscopic techniques have large errors, primarily because the radial velocity of the white dwarf cannot be 
directly measured and must instead be inferred from accretion disk emission lines (see Warner 1990 for a review). Attempts to obtain $M_{\mathrm{WD}}$ from a mass-luminosity relation similar to that for AGB stars are hampered by uncertainties in the distance. However, MFT argue that $T_{\max }$, the maximum effective temperature reached by the remnant before turning-off mainly depends on $M_{\mathrm{WD}}$ and their polytropic model gives a dependence

$$
T_{\max } \approx 6.610^{5}\left(\frac{M_{\mathrm{WD}}}{\mathrm{M}_{\odot}}\right)^{1.6} \mathrm{~K},
$$

which agrees with the detailed stellar models of Fujimoto \& Iben (1992) within $10 \%$. Hence determination of $T_{\max }$ from fitting stellar atmosphere models to the results of a campaign of monitoring classical novae at soft X-ray energies has the potential of determining $M_{\mathrm{WD}}$.

\section{The soft X-ray observations}

The detection of soft X-rays from three classical novae, Nova GQ Mus 1983, Nova PW Vul 1984 and Nova QU Vul 1984, at times ranging from 100 to $1000 \mathrm{~d}$ after optical maximum with the EXOSAT satellite (Ögelman, Beuermann \& Krautter 1984; Ögelman, Krautter \& Beuermann 1987, hereafter $\mathrm{OKB})$, has provided observational verification that the constant bolometric luminosity phase does extend to high effective temperatures $\left(>210^{5} \mathrm{~K}\right)$, as originally suggested by MFT.

Since the majority of the EXOSAT observations were made with only the thin Lexan filter on the low energy telescope, there is, in general, insufficient data in the X-ray measurements alone to discriminate between emission from a hot photosphere and emission from shocked circumstellar gas. OKB also find that the single observation of Nova Vul 1984 \#2 with the Boron filter, $307 \mathrm{~d}$ after optical maximum, is not significant enough to exclude or support a thermal bremsstrahlung spectrum. However, they favor emission from a hot photosphere on the basis of consideration of plasma cooling times, and the absence of coronal [Fe XIV] $\lambda 5303$ lines that should be emitted from shocked gas (Kurtz, Vanden Bout \& Angel 1972). Krautter \& Williams (1989) rule out shocked circumstellar gas by considering the degree and temporal evolution of the ionization of the ejecta of GQ Mus and argue that it is consistent with photo-ionization from a hot radiation source. EXOSAT observations made two months after the 1985 January outburst of the recurrent nova RS Oph (Mason et al. 1987) have been interpreted as due to $\mathrm{X}$-rays from a shock front moving into a circumstellar envelope formed by the pre-outburst wind of the red giant companion (O'Brien, Kahn \& Bode 1987). Even so, the X-ray emission $200 \mathrm{~d}$ after outburst is consistent with an origin on the surface of a white dwarf (Mason et al. 1987). 
The greater energy resolution of the ROSAT PSPC permits a clearer distinction between a hot photosphere and shocked gas. The X-ray emission from GQ Mus in 1992 February, 9 yr after optical maximum, has a very soft spectrum and is consistent with a photospheric origin, at near $\mathrm{L}_{\mathrm{Edd}}$ (Ögelman et al. 1993). ROSAT spectra of Nova Her 1991 (Lloyd et al. 1992), and Nova V1974 Cyg 1992 (Krautter, Ögelman \& Starrfield 1992), 5 and $60 \mathrm{~d}$ after optical maximum respectively, are hard and the implied luminosities are below $10^{-2} L_{\mathrm{Edd}}$, perhaps indicating an origin in shocked gas. GQ Mus was observed twice again with the ROSAT PSPC in 1993 (Shanley et al. 1995). The count rates fell sharply from 1992 February to 1993 January and GQ Mus was not detected in 1993 August/September, indicating that GQ Mus had turned off $10 \mathrm{yr}$ after optical maximum. Nova V1974 Cyg was monitored with ROSAT throughout its active phase in $\mathrm{X}$-rays and was found to turn off 18 months after optical maximum (Starrfield et al. 1995).

In anticipation of these ROSAT detections of classical novae, MacDonald \& Vennes (1991, hereafter MV) performed model atmosphere calculations of the X-ray emission from the photospheres of hot, high gravity stars, using representative values for the white dwarf mass and luminosity of $M_{\mathrm{WD}}=1.2 \mathrm{M}_{\odot}$ and $L_{\mathrm{WD}}=4.210^{4} \mathrm{~L}_{\odot}$. Stellar atmospheres in local thermodynamic equilibrium were calculated for a range of effective temperatures from $10^{5}$ to $10^{6} \mathrm{~K}$ (the corresponding range in $\log g$ is from 4.85 to 8.85) and two compositions representative of the ejecta of CNO novae and ONe novae. An important result of this study is that blackbody fits to the X-ray spectra can grossly underestimate, by as much as a factor of 2, the true effective temperature. Indeed for GQ Mus, Ögelman et al. (1993) found the best blackbody fit temperature of $2.510^{5} \mathrm{~K}$ gave a bolometric flux that was about a factor 100 too high to be consistent with the Eddington limit. However, within the 2 and $3 \sigma$ error limits, they could find a blackbody fit with higher temperature $\left(\simeq 3.510^{5} \mathrm{~K}\right)$ and lower bolometric flux. We estimate that use of MV model atmospheres (see Fig. 3 of MV) would give $T_{\text {eff }}=410^{5} \mathrm{~K}$. Starrfield et al. (1995) also find that the UV colours of GQ Mus from 1987 to 1994 are inconsistent with blackbodies. Furthermore, Krautter \& Starrfield (1995) find that blackbody fits to the V1974 Cyg ROSAT data give super-Eddington fluxes, and that this problem is cured by use of the MV fluxes.

V1974 Cyg was also detected $280 \mathrm{~d}$ after visual maximum by EUVE in the all-sky survey. The count rates were $0.108 \pm 0.010 \mathrm{~s}^{-1}$ and $0.091 \pm$ $0.010 \mathrm{~s}^{-1}$ in the Lexan/Boron and $\mathrm{Al} / \mathrm{C} / \mathrm{Ti}$ filters respectively (Bowyer et al. 1994). A preliminary analysis of these data was given by MacDonald \& Vennes (1994), who found $T_{\text {eff }}$ to be between $310^{5}$ and $3.510^{5} \mathrm{~K}$ and the column density about $1.810^{21} \mathrm{~cm}^{-2}$. Stringfellow \& Bowyer (1994), using 
better calibrated effective area curves for the two filters, find $T_{\text {eff }}=4.0 \pm$ $0.510^{5} \mathrm{~K}$ and $N_{\mathrm{H}} \simeq 310^{21} \mathrm{~cm}^{-2}$. We note that the temperatures found for these two objects and also for QU Vul from analysis of the EXOSAT data by MV, are remarkably similar. Pequignot (1996) has also determined $T_{\text {eff }}$ as a function of time for GQ Mus from photoionization models for the emission lines, assuming a blackbody source of ionizing photons. Although his results depend somewhat on the evolution of the nebular density, he finds that the stellar remnant reaches high temperatures relatively quickly $\left(\simeq 310^{5} \mathrm{~K}\right.$ after one year) and then remains constant or increases slowly over the next $2000 \mathrm{~d}$. The initial relatively rapid temperature increase may indicate that mass loss at a high rate continued even after the stellar remnant was inside its Roche lobe. The long duration of essentially constant temperature is perhaps explained by an episode of accretion as suggested by Ogelman et al. (1993).

Using the above values for $T_{\max }$, our estimate for $M_{\mathrm{WD}}$ is $0.75 \mathrm{M}_{\odot}$ for both stars. Furthermore, to get the short turnoff times requires mass loss at average rates of $810^{-6} \mathrm{M}_{\odot} \mathrm{yr}^{-1}$ for GQ Mus and $510^{-5} \mathrm{M}_{\odot} \mathrm{yr}^{-1}$ for V1974 Cyg. The estimated mass is much lower than thermonuclear runaway theory requires for classical nova outbursts (MacDonald 1983). A possible resolution of this discrepancy is that there is an optically thick outflow from the surface which results in a more extended envelope and lower $T_{\text {eff }}$ than for a hydrostatic envelope. A second possibility is that accretion of near solar composition material before or during the soft X-ray phase covers up the metal-enriched envelope so that the use of the model atmospheres calculated by MV for metal-enriched compositions gives misleading temperature estimates.

\section{References}

Bath, G.T., 1978, MNRAS, 182, 35

Bowyer, S., Lieu, R., Lampton, M., et al., 1994, Ap. J. Supp., 93, 569

Feast, M.W., Glass, I.S., 1974, MNRAS, 167, 81

Fujimoto, M.Y., Iben, I., 1992, AJ, 399, 646

Gallagher, J.S., Code, A.D., 1974, Ap. J., 189, 303

Gallagher, J.S., Starrfield, S., 1976, MNRAS, 176, 3

Gallagher, J.S., Starrfield, S., 1978, ARAA, 16, 171

Geisel, S.L., Kleinmann, D.E., Low, F.J., 1970, Ap. J., 161, L101

Hyland, A.R., Neugebauer, G., 1970, Ap. J.; 160, L177

Kato, M., 1983, PASJ, 35, 507

Kato, M., Hachisu, I., 1994, Ap. J., 437, 802

Krautter, J., Ögelman, H., Starrfield, S., 1992, IAUC 5550

Krautter, J., Starrfield, S., 1995, private communication

Krautter, J., Williams, R.E., 1989, Ap. J., 341, 968

Kurtz, D.W., Vanden Bout, P.A., Angel, J.R.P., 1972, Ap. J., 178, 701

Lloyd, H.M., O’Brien, T.J., Bode, M.F., et al., 1992, Nature, 356, 222

MacDonald, J., 1980, MNRAS, 191, 933 
MacDonald, J., 1983, Ap. J., 267, 732

MacDonald, J., 1984, Ap. J., 283, 241

MacDonald, J., Fujimoto, M.Y., Truran, J.W., 1985, Ap. J., 294, 263

MacDonald, J., Vennes, S., 1991, Ap. J., 373, L51

MacDonald, J., Vennes, S., 1994, AAS meeting Minneapolis, MN, May 29 - June 2

Mason, K.O., Cordova, F.A., Bode, M.F., Barr, P., 1987, in "RS Ophiuchi (1985) and the Recurrent Nova Phenomenon", ed. M.F. Bode, VNU Science Press, Utrecht, p167

O'Brien, T., Kahn, F.D., Bode, M.F., 1987, in "RS Ophiuchi (1985) and the Recurrent Nova Phenomenon", ed. M.F. Bode, VNU Science Press, Utrecht, p177

Ögelman, H., Beuermann, K., Krautter, J., 1984, Ap. J., 287, L31

Ögelman, H., Krautter, J., Beuermann, K., 1987, A\&A, 177, 110

Ögelman, H., Orio, M., Krautter, J., Starrfield, S., 1993, Nature, 361, 331

Paresce, F., Livio, M., Hack, W., Korista, K., 1995, A\&A, 299, 823

Pequignot, D., 1996, these proceedings, p305

Prialnik, D., Shara, M., 1995, AJ, 109, 1735

Prialnik, D., Kovetz, A., 1995, Ap. J., 445, 789

Shanley, L., Ögelman, H., Gallagher, J.S., et al., 1995, Ap. J., 438, L95

Starrfield, S., 1979, in "White Dwarfs and Variable Degenerate Stars", ed. H.M. Van Horn and V. Weidemann, p274

Starrfield, S., Truran, J.W., Sparks, W. M., Kutter, G.S., 1974, Ap. J., 176, 169

Starrfield, S., Shore, S.N., Sparks, W.M., et al., 1992, Ap. J., 391, L71

Starrfield, S., et al., 1996, in IAU Colloquium 152, "Astrophysics in the Extreme Ultraviolet", Cambridge University Press, Cambridge, in press

Stringfellow, S.G., Bowyer, S., 1994, in "Interacting Binary Stars", ed. A. Shafter, ASP Conf. Ser. 56, 315

Warner, B., 1990 in IAU Colloquium 122, "Physics of Classical Novae", eds A. Cassatella, R. Viotti, Springer-Verlag, Berlin, p24 